\title{
Surface Photograft Polymerization of Vinyl Acetate on Low Density Polyethylene Film. Effects of Solvent
}

\author{
Jian-Ping DENG, Wan-Tai YANG, ${ }^{\dagger}$ and Bengt RANBY ${ }^{*}$ \\ Department of Polymer Science, Beijing University of Chemical Technology, Beijing, 100029, China \\ ${ }^{*}$ Department of Polymer Technology, Royal Institute of Technology, S-10044 Stockholm, Sweden
}

(Received March 6, 2000; Accepted June 20, 2000)

\begin{abstract}
To photograft vinyl acetate (VAc) onto low density polyethylene (LDPE) film, suitable solvents should be determined in advance. Different reagents were used as solvents of VAc (acetone, benzene, carbon tetrachloride, cyclohexane, cyclohexanone, ethyl acetate, methanol, tetrahydrofuran (THF) and $N, N$-dimethylformamide(DMF). All graft polymerizations were completed by two-steps, i.e., benzophenone (BP) pre-coated film samples were prepared onto which VAc was grafted. To prepare film samples containing BP, BP should be dissolved in a suitable solvent. The effects of various solvents of BP on grafting were examined, including acetone, benzene, chloroform, 2,4-dioxane, DMF, ethyl acetate, $n$-hexane, methanol and THF. Percent conversion (CP), grafting efficiency (GE) and grafting percent (GP) determined by gravimetry and contact angles of grafted films against water showed that reagents have different effects on the graft polymerizations. Acetone is the most suitable as solvent of BP or VAc. The behavior of solvents seems closely related to the affinity with the substrate.

KEY WORDS Photograft Polymerization / Low Density Polyethylene Film / Vinyl Acetate / Benzophenone / Solvent /
\end{abstract}

In our previous papers, ${ }^{1,2}$ VAc was photografted onto LDPE film with different photoinitiators, and graft polymerization was verified with FT-IR spectra. Among photoinitiators, BP is preferred. The main grafting mechanism can be briefly outlined here. When irradiated with UV radiation, BP absorbs energy and is excited to singlet state $[\mathrm{BP}]^{\mathrm{S}}$ and then relaxes to more stable triplet state $[\mathrm{BP}]^{\mathrm{T}}$. $[\mathrm{BP}]^{\mathrm{T}}$ abstracts hydrogens from the chains of LDPE, leading to LDPE macromolecular free radicals generated. Adding monomer makes graft polymerization take place. BP pre-coating is the most suitable. ${ }^{3}$ To prepare BP pre-coated film samples, BP should be dissolved in a suitable solvent. Reagents having different solubility parameters (SP) may show different diffusion behavior on film. Therefore, it is important to determine suitable solvents of BP. However, no studies have focused on this in the literatures. Some papers study the effects of solvents of monomer on graft polymerization. ${ }^{4-6}$ From these studies, it is clear that affinity between substrates and monomers is a decisive factor for effective grafting. Swelling of the film caused by the solvents also has great influence on the distribution and average molecular weights of grafted chains.

The present paper centers on the effects of various solvents of BP and VAc on the photograft polymerization. Emphasis is laid on the solubility parameters of solvents to explain their behaviors.

\section{EXPERIMENTAL}

\section{Materials}

Monomer: Vinyl acetate (VAc, AR grade, Bp: 72-73 ${ }^{\circ} \mathrm{C}$, from Tianjin Tiantai Chemical Reagent Plant, China), was purified by distillation before use.

Photoinitiator: Benzophenone (BP, chemically pure grade, mp: $47-49^{\circ} \mathrm{C}$, from Shanghai Reagent Plant No.1, China), was used as received.

\footnotetext{
${ }^{\dagger}$ To whom correspondence should be addressed.
}

LDPE film: Commercial LDPE film $(63 \mu \mathrm{m}$ in thickness, transparency to UV light, $75 \%, \lambda=254 \mathrm{~nm}$ ), was cut into circular samples with diameter of $70 \mathrm{~mm}$, and subjected to Soxhlet extraction with acetone for $5 \mathrm{~h}$ to remove additives and impurities.

Solvent: Acetone, benzene, carbontetrachloride, chloroform, cyclohexane, cyclohexanone, $N, N$-dimethylform amide (DMF), 2, 4-dioxane, ethyl acetate, $n$-hexane, methanol, and tetrahydrofuran (THF), all analytically pure grade, were used without further purification.

\section{Preparing BP Pre-Coated Films}

BP pre-coated film samples were prepared as described earlier. ${ }^{3}$ A drop of acetone (unless otherwise noted) solution containing a known amount of $\mathrm{BP}$ was placed between two film samples with a micro-syringe. A certain pressure was exerted to make the solution thin and even. The samples were separated and dried at ambient temperature and BP pre-coated film samples were obtained. The amount of BP on each sample was controlled by the volume of $\mathrm{BP}$ solution.

\section{Grafting Procedure}

The equipment for photografting and assembly of film samples for polymerization were introduced in detail in the previous papers. ${ }^{1,2} \mathrm{~A}$ certain amount of monomer solution (pre-purged with nitrogen gas to exclude oxygen dissolved in the solution) was placed between two films with a micro-syringe. A appropriate pressure was given to make the reaction liquid thin and even. The system was covered with a piece of quartz plate, laid on the holder of the irradiation equipment at a distance about $15 \mathrm{~cm}$ from the UV lamp, and irradiated by UV radiation (UV lamp: high-pressure mercury lamp, $1000 \mathrm{w}$ ) at a given temperature controlled by a thermocouple thermometer. Polymerization was decided by a predetermined period of irradiation time.

When effects of solvents of BP on grafting were investigated, different solvents containing the equal amount 
of BP were used to prepare BP pre-coated film samples as substrates. Neat VAc was used as monomer. When effects of the solvents of VAc on grafting were studied, acetone solution containing given amount of BP was used to prepare BP pre-coated film samples. The samples were used as substrates and solutions of VAc $\left(5 \mathrm{~mol} \mathrm{~L}^{-1}\right)$ in different solvents were used as monomer solutions.

After grafting, the samples were dried to constant weight and subjected to Soxhlet extraction with suitable solvents for $8 \mathrm{~h}$ to remove the homopolymer. Three grafting parameters, percent conversion (CP), grafting efficiency (GE) and grafting percent (GP) were calculated as follows:

$$
\begin{aligned}
& \mathrm{CP}=\frac{W_{\mathrm{P}}}{W_{\mathrm{M}}} \times 100 \% \\
& \mathrm{GE}=\frac{W_{\mathrm{G}}}{W_{\mathrm{P}}} \times 100 \% \\
& \mathrm{GP}=\frac{W_{\mathrm{P}}}{W_{\mathrm{F}}} \times 100 \%
\end{aligned}
$$

Where $W_{M}$ is weight of monomer between the two films; $W_{\mathrm{P}}$ is weight of polymer formed, including homopolymer and grafted polymer, obtained by weighing the films after vaporizing residual monomer; $W_{G}$ is weight of the grafted polymer, obtained after extracting the homopolymer with acetone; and $W_{F}$ is the weight of LDPE film before grafting.

\section{Alcoholysis and Measuring Contact Angles}

By alcoholysis as reported earlier, ${ }^{2}$ PE-g-PVAc was turned into PE-g-PVA. Contact angles of PE-g-PVA film samples against water were measured, as described in another paper. ${ }^{2}$

\section{RESULTS AND DISCUSSION}

\section{Different Solvents of $B P$}

Various reagents were applied as solvents of BP including acetone, methanol, ethyl acetate, dioxane, chloroform, $n$-hexane, THF, DMF and benzene, whose effects on graft polymerizaton of VAc are presented in Table I.

Nine reagents with different behavior, which may be related closely to solubility parameters (SP) listed in Table II, were used.

Generally, if SP of one reagent is closer to that of PE, it is easier for the reagent to diffuse through $\mathrm{PE}$ film. Chloroform, though its SP is around 9.4, has behavior similar to that of $n$-hexane. Methanol, whose SP is about 14.8, diffuses on PE films much more smoothly than $\mathrm{DMF}(\mathrm{SP}=12.0$ ). According to SPs and behavior, the reagents are grouped as having higher affinity with $\mathrm{PE}$ film samples, such as $n$-hexane and chloroform; having medium affinity, including acetone, methanol, dioxane, ethyl acetate, THF and benzene; and having lower affinity, e.g., DMF. Mainly owing to different affinities, the results in Table I were obtained. Their behavior is illustrated in the following Figures $1 \mathrm{~A}-1 \mathrm{C}$.

\begin{tabular}{|c|c|c|c|c|c|}
\hline Solvent & $\mathrm{BP} / \mathrm{VAc} / \mathrm{wt} \%$ & $\mathrm{CP} / \%$ & $\mathrm{GE} / \%$ & GP/\% & $\frac{\text { Contact angle }^{a, t}}{\text { degree }}$ \\
\hline$n$-Hexane & 1 & 9.3 & 2.0 & 0.05 & 91 \\
\hline$n$-Hexane & 3 & 18.7 & 10.1 & 0.11 & 80 \\
\hline n-Hexane & 5 & 28.0 & 28.3 & 0.45 & 75 \\
\hline Chloroform & 1 & 14.9 & 1.1 & 0.05 & 92 \\
\hline Chloroform & 3 & 24.3 & 7.6 & 0.11 & 85 \\
\hline Chloroform & 5 & 29.9 & 28.1 & 0.48 & 78 \\
\hline Acetone & 1 & 22.4 & 16.7 & 0.21 & 81 \\
\hline Acetone & 3 & 46.7 & 46.3 & 1.23 & 72 \\
\hline Acetone & 5 & 59.8 & 80.4 & 2.74 & 56 \\
\hline Methanol & 1 & 20.8 & 13.5 & 0.16 & 79 \\
\hline Methanol & 3 & 36.4 & 44.5 & 0.92 & 71 \\
\hline Methanol & 5 & 59.2 & 62.5 & 2.11 & 65 \\
\hline Dioxane & 1 & 19.6 & 12.8 & 0.14 & 80 \\
\hline Dioxane & 3 & 37.4 & 32.5 & 0.69 & 76 \\
\hline Dioxane & 5 & 53.5 & 59.0 & 1.80 & 64 \\
\hline Ethyl acetate & 1 & 28.9 & 17.5 & 0.29 & 80 \\
\hline Ethyl acetate & 3 & 35.5 & 50.0 & 1.01 & 72 \\
\hline Ethyl acetate & 5 & 52.1 & 71.8 & 2.13 & 60 \\
\hline THF & 1 & 19.6 & 8.1 & 0.09 & 82 \\
\hline THF & 3 & 31.5 & 28.6 & 0.51 & 73 \\
\hline $\mathrm{THF}$ & 5 & 51.8 & 57.5 & 1.70 & 66 \\
\hline Benzene & 1 & 15.9 & 10.0 & 0.15 & 85 \\
\hline Benzene & 3 & 31.5 & 50.1 & 0.90 & 73 \\
\hline Benzene & 5 & 55.2 & 61.5 & 1.93 & 63 \\
\hline DMF & 1 & 24.3 & 1.1 & 0.01 & 92 \\
\hline $\mathrm{DMF}$ & 3 & 29.9 & 25.6 & 0.40 & 72 \\
\hline DMF & 5 & 36.4 & 32.8 & 0.62 & 61 \\
\hline
\end{tabular}

$n$-Hexane and chloroform (Figure 1A) as solvents of
Table I. Effects of solvents of BP on polymerization

${ }^{\text {a }}$ Contact angle of PE film with water before grafting at $94^{\circ}$.

${ }^{b}$ Contact angle of inner side of sample; polymerization conditions: volume of VAc, $25 \mu \mathrm{L}$; intensity of UV radiation, $5010 \mu \mathrm{w} \mathrm{cm} \mathrm{cm}^{-2}$; temperature, $30^{\circ} \mathrm{C}$; polymerization time, $4 \mathrm{~min}$.

Table II. Solubility parameters of reagents ${ }^{7}$

\begin{tabular}{lc}
\hline \multicolumn{1}{c}{ Reagent } & Solubility parameter(SP) \\
\hline$n$-Hexane & 7.2 \\
LDPE $^{\mathrm{a}}$ & 7.9 \\
Cyclohexane & 8.18 \\
Carbon tetrachloride & 8.6 \\
Vinyl acetate(VAc) & 9.0 \\
Ethyl acetate & 9.1 \\
Benzene & 9.15 \\
THF & 9.2 \\
Chloroform & 9.4 \\
Acetone & 9.8 \\
2,4-Dioxane & 10.1 \\
DMF & 12.0 \\
Methanol & 14.8 \\
\hline
\end{tabular}

${ }^{\mathrm{a}}$ Low density polyethylene.

$\mathrm{BP}$, they penetrate $\mathrm{PE}$ films rapidly, carrying $\mathrm{BP}$ molecules. When VAc is added, VAc cannot penetrate PE films at the same velocity as $n$-hexane and chloroform. Therefore, VAc and BP cannot contact effectively.

For acetone, methanol, THF, dioxane, ethyl acetate and benzene (Figure 1B), affinity with PE film is the same as that of VAc. So VAc molecules contact with BP molecules effectively. Polymerization of these systems thus proceeds smoothly.

The affinity of DMF with PE film is lowest (Figure 1C). When deposited between the two films, DMF concentrates mostly on the inner surface (F1 and F2, in Figure 2). When VAc is deposited, VAc carries BP along to the deeper layers of the film, making them sufficiently contact. Polymerization proceeds more smoothly than that of the system using $n$-hexane and chloroform as the solvents of BP, but less smoothly than those applying 


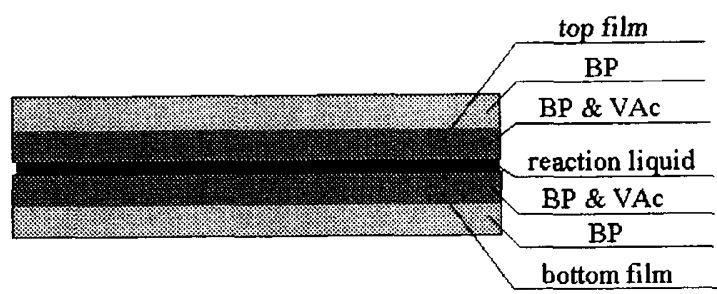

B

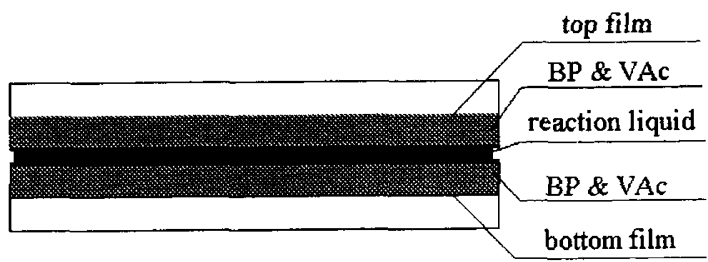

$\mathrm{C}$

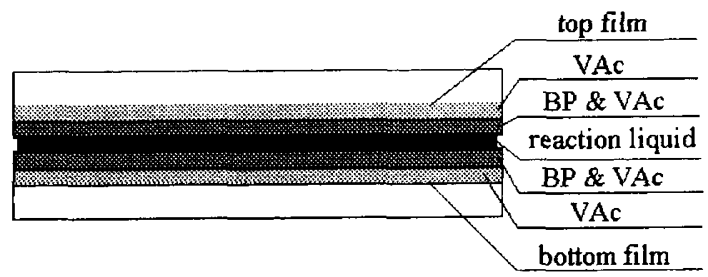

Figure 1. Effects of affinity of the solvents with PE films on grafting polymerization.

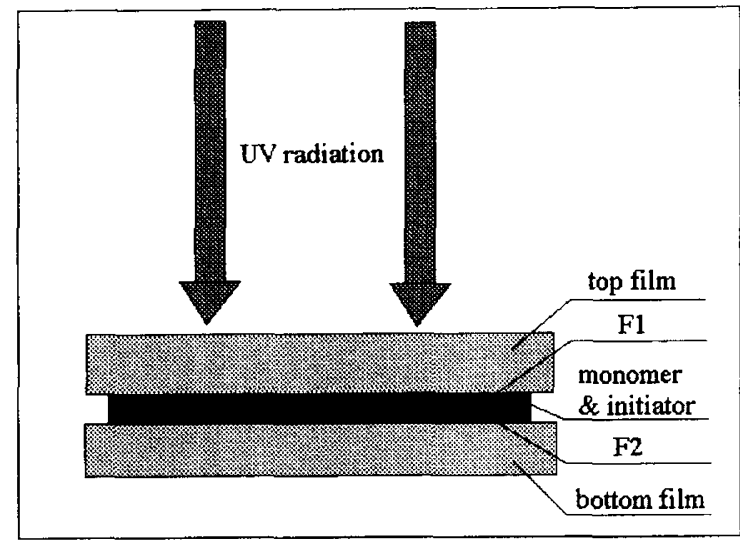

Figure 2. Cross section of grafting polymerization.

acetone.

The data in Table I demonstrate that contact angles of the grafted films with water decline with increase of GP, irrespective of solvent. The explanation above can be verified indirectly to some extent by contact angles of grafted PE films (Table I). For the first group of solvents, contact angles decrease slowly, probably due to the low quantity of GP and deep penetration of the grafted chains. Regarding the third group, although all CP, GE, and GP of the polymerization systems are at a lower level compared with those of the second group, contact angles fall rapidly, indicating graft polymerization mostly takes place near the surface layer. For the second group, due to total effects of much more VAc grafted and more grafted PVAc chains diffusing on the surface regions of the film, the drop in contact angles is more considerable. Contact angles are influenced by several factors. From the above, solvent of BP acts just as a carrier, diffusing $\mathrm{BP}$ on the film. If the affinity between the sol-
Table III. Effects of solvents of VAc on polymerization

\begin{tabular}{|c|c|c|c|c|c|}
\hline Solvent & $\begin{array}{l}\text { Irradiation } \\
\text { time/min }\end{array}$ & $\mathrm{CP} / \%$ & $\mathrm{GE} / \%$ & $\mathrm{GP} / \%$ & $\frac{\text { Contact angle }}{\text { degree }}$ \\
\hline Acetone & 1 & 25.8 & 26.7 & 0.18 & 80 \\
\hline Acetone & 3 & 45.3 & 50.1 & 0.59 & 71 \\
\hline Acetone & 5 & 69.8 & 79.9 & 1.46 & 63 \\
\hline Cyclohexanone & 1 & 25.5 & 25.0 & 0.17 & 81 \\
\hline Cyclohexanone & 3 & 37.8 & 34.2 & 0.33 & 75 \\
\hline Cyclohexanone & 5 & 41.1 & 65.1 & 0.70 & 71 \\
\hline Methanol & 1 & 26.7 & 26.7 & 0.19 & 78 \\
\hline Methanol & 3 & 48.8 & 58.8 & 0.75 & 73 \\
\hline Methanol & 5 & 54.7 & 64.7 & 0.93 & 65 \\
\hline Ethyl acetate & 1 & 22.8 & 37.5 & 0.22 & 80 \\
\hline Ethyl acetate & 3 & 45.5 & 50.0 & 0.60 & 70 \\
\hline Ethyl acetate & 5 & 56.4 & 63.3 & 0.94 & 63 \\
\hline Carbon tetrachloride & 1 & 18.9 & 3.1 & 0.02 & 93 \\
\hline Carbon tetrachloride & 3 & 35.5 & 16.1 & 0.15 & 87 \\
\hline Carbon tetrachloride & 5 & 41.4 & 32.8 & 0.35 & 80 \\
\hline Cyclohexane & 1 & 5.6 & 8.1 & 0.01 & 92 \\
\hline Cyclohexane & 3 & 20.5 & 40.0 & 0.22 & 83 \\
\hline Cyclohexane & 5 & 32.7 & 41.4 & 0.35 & 75 \\
\hline THF & 1 & 19.6 & 8.1 & 0.04 & 84 \\
\hline THF & 3 & 41.5 & 38.6 & 0.42 & 78 \\
\hline $\mathrm{THF}$ & 5 & 60.8 & 61.5 & 0.98 & 67 \\
\hline DMF & 1 & 24.3 & 1.2 & 0.01 & 91 \\
\hline DMF & 3 & 29.9 & 25.6 & 0.20 & 73 \\
\hline DMF & 5 & 36.4 & 42.8 & 0.41 & 60 \\
\hline Benzene & 1 & 7.3 & 10.6 & 0.02 & 85 \\
\hline Benzene & 3 & 9.8 & 47.8 & 0.12 & 74 \\
\hline Benzene & 5 & 11.6 & 51.8 & 0.16 & 62 \\
\hline
\end{tabular}

${ }^{\mathrm{a}}$ Contact angle of PE film before grafting at $94^{\circ}$. ${ }^{\mathrm{b}}$ Contact angle of inner side of PE film; polymerization conditions: content of $\mathrm{BP}, 3 \mathrm{wt} \%$ of VAc; volume of VAc, $25 \mu \mathrm{L}$; polymerization temperature, $30^{\circ} \mathrm{C}$; intensity of UV radiation, $5110 \mu \mathrm{W} \mathrm{cm}{ }^{-2}$.

vent and LDPE film is too high, the effective amount of $\mathrm{BP}$ becomes limited (hexane and chloroform); if the affinity is too low, the active hydrogens abstracted by $[\mathrm{BP}]^{\mathrm{T}}$ are limited (DMF). Therefore, only those reagents with moderate affinity to LDPE film are effective.

\section{Different Solvents of VAc}

Various reagents were applied as solvents of VAc, including acetone, cyclohexanone, cyclohexane, methanol, ethyl acetate, carbon tetrachloride, tetrahydrofuran (THF), $N, N$-dimethylformamide (DMF), and benzene. The results are listed in Table III.

From CP, GE, and GP in Table III, it seems that the behavior of these different solvents is related to solubility parameter (SP), absorbing of UV radiation and reactivity and initiating ability of the solvent.

\section{Solubility Parameter (SP)}

According to the solubility parameters (SP, Table II), these reagents can be classified as, carbon tetrachloride and cyclohexane have higher affinity to LDPE film; acetone, methanol, ethyl acetate, THF and benzene have medium affinity; and DMF has lower affinity. The different affinities lead to different influence on graft polymerization.

The swelling of film samples caused by solvents is probably another factor of graft polymerization. $\mathrm{H}$. Kubota $^{6}$ investigated the influence of swelling on grafting vinyl monomers onto cellulose. In this study, several reagents whose SPs are similar to that of PE film help to swell the film, which is favorable for VAc to penetrate into the deeper layers of the film. In our earlier study, ${ }^{3}$ pre-irradiation was used to complete grafting VAc onto 
Table IV. Molar extinction coefficients of reagents

\begin{tabular}{llcc}
\hline \multicolumn{1}{c}{ Reagent } & Solvent & $\begin{array}{c}\text { Maximum } \\
\text { wavelength/nm }\end{array}$ & $\begin{array}{c}\text { Molar extinction } \\
\text { coefficient (log } \varepsilon \text { ) }\end{array}$ \\
\hline Benzene & Oil ether & 255 & 2.35 \\
Acetone & Ethanol & 271 & 1.20 \\
Acetone & Cyclohexane & 270 & 1.99 \\
Cyclohexanone & Hexane & 285 & 1.15 \\
Ethyl acetate & Cyclohexane & 220 & 1.69 \\
Benzophenone & Chloroform & 254 & 4.18 \\
\hline
\end{tabular}

LDPE film, using DMF and ethyl acetate as solvents, respectively. Under the same conditions, graft polymerization proceeds more smoothly in ethyl acetate than in DMF. The SP of ethyl acetate $(\mathrm{SP}=9.1)$ is much closer to that of LDPE $(\mathrm{SP}=7.9$ ) than that of DMF ( $\mathrm{SP}=12.0$ ). Accordingly, it is easier for ethyl acetate to diffuse through LDPE film than DMF. During diffusion, ethyl acetate makes LDPE film swell to a certain degree, which is favorable to graft polymerization.

\section{Absorbing of UV Radiation}

As solvents of VAc, acetone, cyclohexanone, ethyl acetate, DMF and benzene absorb UV radiation (200-400 $\mathrm{nm})$ at certain wavelengths. The molar extinction coefficients $(\varepsilon)$ and maximum absorption wavelengths of these reagents are presented in Table IV.

Although the extinction coefficients are negligible compared with that of $\mathrm{BP}\left(\varepsilon=17000 \mathrm{~L} \mathrm{~mol}^{-1} \mathrm{~cm}^{-1}, \lambda=\right.$ $254 \mathrm{~nm}$ ), their amounts are much more than that of BP. Therefore, absorbing UV radiation by these solvents is probably important for graft polymerization. Table III shows that benzene is not a suitable solvent of VAc. When benzene is applied as solvent of VAc, polymerization proceeds with much difficulty. When the reaction system is irradiated for $5 \mathrm{~min}$, only $11.6 \%$ of VAc takes part in polymerization, of which about $51.8 \%$ is grafted polymer. It seems closely related to the absorption of UV radiation by benzene at around $255 \mathrm{~nm}$. Based on our previous research, ${ }^{2}$ when far UV radiation $(200-300$ $\mathrm{nm}$ ) is excluded with a piece of polyethylene terephthalate (PET) film, the graft polymerization of VAc on LDPE film is restrained greatly. Consequently, the strong absorption of UV radiation at $255 \mathrm{~nm}$ by benzene ( $\log \varepsilon=2.35$, Table IV) may lower the initiating efficiency of BP. Graft polymerization proceeds just on the surface of the film because the screen of UV radiation by benzene makes the reaction difficult in the deep layers. As a result, the grafted chains diffuse mainly on surface regions, and are confirmed indirectly by the contact angles in Table III.

\section{Reactivity and Initiating Ability}

Considering the initiation mechanism of $\mathrm{BP},{ }^{1}$ it should be noted that in polymerization systems, solvents containing active hydrogens should be avoided. In Tazuke and colleagues' article, ${ }^{8}$ it is thought that methanol, as solvent, inhibits graft polymerization; but in Davis' studies, ${ }^{4}$ methanol was used as solvent to graft styrene on cellulose. It is obvious from the data in Table III that methanol affects graft polymerization slightly. The reason may be that the bond energy of $\mathrm{H}-\mathrm{CH}_{2} \mathrm{OH}$ is so high that it is not easy for $[\mathrm{BP}]^{\mathrm{T}}$ to abstract hydrogens from $\mathrm{CH}_{3} \mathrm{OH}^{9}$

According to Table III, DMF is not suitable as solvent of VAc, which is related closely to the fact that it cannot penetrate through $\mathrm{PE}$ films. Pashova ${ }^{10}$ considers that DMF is an undesirable component of the solvent for polymerization, because nitrogen atoms on DMF may participate in the interactions with primary radicals formed when BP is irradiated by UV radiation.

It is thus concluded that acetone is the most useful solvent in graft polymerization, in agreement with the results by Tazuke and colleagues. ${ }^{11}$ In their opinion, acetone has high stability against hydrogen abstraction by excited sensitizers. The ability of acetone to initiate graft polymerization when irradiated by UV radiation may make contribution to it as mentioned by Allmer. ${ }^{12}$

Acknowledgment. This research was supported by the Chinese National Nature Science Foundation, which is gratefully acknowledged.

\section{REFERENCES}

1. J. P. Deng, W. T. Yang, and B. Rånby, J. Appl. Polym. Sci., in press.

2. J. P. Deng, W. T. Yang, and B. Rånby, J. Appl. Polym. Sci., in press.

3. J. P. Deng, W. T. Yang, and B. Rånby, J. Appl. Polym. Sci., in press.

4. N. P. Davis, J. L. Garnett, and R. Urquhart, J. Polym. Sci., Polym. Lett. Ed., 14, 537 (1976).

5. Y. Ogiwara, K. Torikoshi, and H. Kubota, J. Polym. Sci., Polym. Lett. Ed., 20, 17 (1982).

6. H. Kubota, Y. Murata, and Y. Ogiwara, J. Polym. Sci., Polym. Chem. Ed., 11, 485 (1973).

7. Q. C. Gu, S. C. Lou, and Q. P. Dai, in "Chemistry Handbook", Hua Xue Yong Biao, Ed., Jiangsu Science Technology Press, Nanjing, 1979, pp 5-26.

8. S. Tazuke and H. Kimura, J. Polym. Sci. Polym. Lett., 497 (1978).

9. W. 'T. Yang and B. Rånby, Polym. Bull., 37, 89 (1996).

10. V. S. Pashova, G. S. Georgiev, and V. A. Dakov, J. Appl. Polym. Sci., 51, 807 (1994).

11. S. Tazuke and H. Kimura, Makromol. Chem., 179, 2603 (1978).

12. K. Allmer, A. Hult, and B. Rånby, J. Polym. Sci., Part A: Polym. Chem., 26, 2099 (1988). 